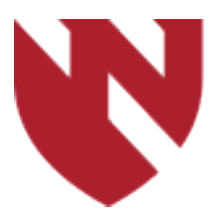

December 2019

\title{
Synthetic Resorbable vs. Cellulose Bandage for Minor Hemorrhage in a Porcine Model
}

\author{
Ujwal R. Yanala \\ University of Nebraska Medical Center \\ Sandra Noriega \\ University of Nebraska Medical Center \\ Ruben Spretz \\ University of Nebraska Medical Center \\ Jorge Ragusa \\ University of Nebraska Medical Center \\ Luis Nunez \\ University of Nebraska Medical Center
}

See next page for additional authors

Tell us how you used this information in this short survey.

Follow this and additional works at: https://digitalcommons.unmc.edu/gmerj

Part of the Higher Education Commons, and the Medicine and Health Sciences Commons

\section{Recommended Citation}

Yanala, U. R., Noriega, S., Spretz, R., Ragusa, J., Nunez, L., Larsen, G., , Carlson, M. Synthetic Resorbable vs. Cellulose Bandage for Minor Hemorrhage in a Porcine Model. Graduate Medical Education Research Journal. 2019 Dec 13; 1(1). https://digitalcommons.unmc.edu/gmerj/vol1/iss1/16 


\section{Synthetic Resorbable vs. Cellulose Bandage for Minor Hemorrhage in a Porcine Model}

\section{Creative Commons License}

\section{(c) (1) $\Theta(9$}

This work is licensed under a Creative Commons Attribution-Noncommercial-No Derivative Works 4.0 License.

\section{Authors}

Ujwal R. Yanala, Sandra Noriega, Ruben Spretz, Jorge Ragusa, Luis Nunez, Gustavo Larsen, and Mark Carlson 
A Comparative Analysis of Survival and Funding Discrepancies in Cancers with High Mortality

Bradley R Hall, Andrew Cannon, Pranita Atri, Christopher S Wichman, Lynette M Smith, Sushil Kumar, Surinder K Batra, Hongmei Wang, Apar Kishor Ganti, Aaron R Sasson, Chandrakanth Are

\section{Mentor: Chandrakanth Are \\ Program: General Surgery}

Background \& Objectives: Discrepancies in research funding may contribute to stagnant survival rates in pancreatic ductal adenocarcinoma (PDAC). Comparative analyses of survival and funding statistics in cancers with high mortality were performed to quantify discrepancies and identify areas for intervention.

Methods: The Surveillance, Epidemiology, and End Results database was queried for survival statistics. Funding data were obtained from the National Cancer Institute (NCI). Clinical trial data were obtained from www. clinicaltrials.gov. Cancers with high mortality were included for analyses.

Results: Since 1997, PDAC has received lesser funding ( $\$ 1.13$ billion) than other cancers such as breast ( $\$ 9.46$ billion), prostate ( $\$ 4.46$ billion), lung ( $\$ 4.26$ billion), and colorectal ( $\$ 4.08$ billion). Similarly, fewer clinical trials have been completed in PDAC $(n=680)$ compared to breast $(n=2,077)$, lung $(n=2,046)$, prostate $(n=1,134)$ and colorectal $(\mathrm{n}=1,196)$ cancer. Despite this, since 1997, NCI dollars invested in PDAC research produced a greater return on investment with regards to 5-year overall survival (5YOS) compared to breast, prostate, uterine, melanoma, and ovarian cancer. Incremental cost effectiveness analysis demonstrates that millions (liver, non-Hodgkin's lymphoma) and billions (colorectal, lung) of dollars were required for each additional $1 \%$ increase in 5Y-OS compared to PDAC. Funding of research towards early detection and diagnosis of PDAC has decreased by $50 \%$ since 2007 . For nearly all cancers, treatment-related research receives the highest percentage of NCI funding.

Conclusions: Funding of PDAC research is significantly less than other cancers despite its higher mortality and greater potential to improve 5Y-OS. Increased awareness and lobbying are required to increase funding, promote research and improve survival.

https://doi.org/10.32873/unmc.dc.gmerj.1.1.015

\section{Synthetic Resorbable vs. Cellulose Bandage for Minor Hemorrhage in a Porcine Model}

Ujwal Yanala, Sandra Noriega, Ruben Spretz, Jorge Ragusa, Luis Nunez, Gustavo Larsen, Mark Carlson

Mentor: Mark Carlson

Program: General Surgery

Introduction: Commercially-available topical hemostats for minor hemorrhage incurred during elective surgical procedures are relatively expensive. We believe that more economical synthetic hemostats could be produced. Our objective here was to compare the efficacy and toxicity of a synthetic resorbable hemostatic bandage vs. an analogous commercial product in a porcine model of minor hemorrhage.

Methods: For the nonsurvival efficacy study, anesthetized domestic swine (boars, 3 months, 29-40 kg) underwent arterial/ venous line placement and splenectomy. A 1 $\mathrm{x} 8 \mathrm{~cm}$ section of liver was resected from the edge of the left lateral lobe, and test bandage (macroporous polycaprolactone mesh, PCL; $\mathrm{N}=10$ ) or oxidized regenerated cellulose (ORC; Surgicel ${ }^{\circledR}$, Ethicon $\AA ; \mathrm{N}=10$ ) was applied with manual pressure for 5 minutes. Resuscitation then was performed with warm LR (target MAP $=80 \%$ of preinjury), and blood loss was measured $60 \mathrm{~min}$ after injury. For the survival toxicity study, a similar resection technique was employed ( $\mathrm{N}=6$ for each material), and necropsy was performed at 30 days to evaluate for bandage toxicity (subject growth, serum chemistry, histology).

Results: Pre-injury weight, VS, and laboratory testing did not differ among groups. Resection mortality was zero. In the efficacy study, there were no differences between the PCL vs. ORC groups in blood loss or other post-injury variables (Table), except that the resuscitation fluid volume in the ORC group was greater. Other results from the efficacy study not shown in the Table include platelet counts and coagulation testing (no significant differences). Other than minor granuloma formation at the implantation site with both PCL and ORC, the survival study did not reveal any measurable toxicity.

Conclusion: The efficacy and toxicity of the PCL test bandage vs. the ORC comparator were not different in a porcine model of minor hepatic hemorrhage. Based on projected costs of production (not shown), the PCL bandage could represent a lower-cost alternative to $\mathrm{ORC}$ for the treatment of minor surgical bleeding.

https://doi.org/10.32873/unmc.dc.gmerj.1.1.016

\section{Renal Sympathetic Vasomotion Monitoring as a Novel Method for Intraprocedural Feedback for Renal Denervation \\ Peter Ricci Pellegrino, Alicia M. Schiller, Han-jun Wang, Yiannis S. Chatzizisis, Irving H. Zucker}

Mentor: Irving H. Zucker

Program: Anesthesiology

Background \& Objectives: The sympathetic nervous system is a master regulator of homeostasis, and sympathetic dysfunction is implicated in the pathophysiology of cardiovascular, renal, and neurological disease. Despite its widespread importance, sympathetic nervous system outflow cannot be assessed in a clinically useful way, negatively impacting the assessment and treatment of prevalent diseases. One such example is the controversial pivotal trial failure of renal denervation, a promising intervention- based therapy for hypertension in which the renal sympathetic nerves are ablated by an endovascular approach. The inability to assess the sympathetic nervous system, and thus adequate renal sympathetic nerve ablation, remains an existential problem facing the field of renal denervation.

Methods: Based on the fact that renal sympathetic nerve activity exerts rhythmic, baroreflex-driven, and vasoconstrictive control of the renal vasculature, we developed a novel technique for identifying rhythmic sympathetic vascular control using a timevarying, two-component Windkessel model of the renal circulation. This technology was tested in two different animal models of renal denervation; ten rabbits underwent chronic, surgical renal denervation, and nine pigs underwent acute, functional renal denervation via intrathecal administration of ropivacaine.

Results: Both methods of renal denervation reduced low-admittance gain, negativephase shift renal vascular control at known sympathetic vasomotor frequencies, consistent with a reduction in vasoconstrictive, baroreflex-driven renal sympathetic vasomotion, but did not affect mean renal blood flow. 